\title{
EFFECT OF CRACK BRIDGING ON THE TOUGHENING OF CERAMIC/GRAPHENE COMPOSITES
}

\author{
S.V. Bobylev ${ }^{1,2}$ and A.G. Sheinerman ${ }^{1,2,3}$ \\ ${ }^{1}$ Institute of Problems of Mechanical Engineering, Russian Academy of Sciences, \\ St. Petersburg 199178, Russia \\ ${ }^{2}$ Peter the Great St. Petersburg Polytechnic University, St. Petersburg 195251, Russia \\ ${ }^{3}$ Saint Petersburg State University, 7/9 Universitetskaya nab., St. Petersburg 199034, Russia
}

Received: May 30, 2018

\begin{abstract}
A model is proposed describing the effect of crack bridging on the fracture toughness of ceramic/graphene composites. The dependences of the fracture toughness on the graphene content and the sizes of the graphene platelets are calculated in the exemplary case of yttria stabilized zirconia (YSZ)/graphene composites. The calculations predict that if crack bridging prevails over crack deflection during crack growth, the maximum toughening can be achieved in the case of long graphene platelets provided that the latter do not rupture and adhere well to the matrix. The model shows good correlation with the experimental data at low graphene concentrations.
\end{abstract}

\section{INTRODUCTION}

Due to its superior mechanical, optical and thermal properties, high electrical conductivity and a large specific surface area, graphene is an excellent nanofiller in polymer-, metal-, and ceramic-matrix composites (see, e.g., reviews [1-12]). In particular, in the last few years, various research groups have begun synthesizing ceramic nanocomposites containing nanoinclusions (with a thickness of several nanometers) of graphene or reduced graphene oxide [3-5,10-36]. The studies of these composites have shown that graphene inclusions (also called graphene platelets or graphene nanoplates (GNPs)), which are usually located along the grain boundaries of the ceramic matrix, can lead to a significant increase in the fracture toughness, bending strength and electrical conductivity of ceramics [3-5,10-36]. In particular, small volume fractions of nanoplates of graphene or reduced graphene oxide can lead to a significant increase in the fracture toughness of ceramics. For example, the authors of [15] found an increase in the fracture toughness of silicon nitride by 135 percent by adding 1.5 volume percent of GNPs. Such a dramatic increase in fracture toughness was explained [15] primarily by the formation of dense ensembles of graphene sheets that surround individual grains and cause a change in the direction of growing cracks. Lee et al. [25] observed a 2.5-fold increase of the fracture toughness of alumina after the addition of 2 vol.\% of reduced graphene oxide. Another example of a significant increase in fracture toughness is tantalum carbide [22], in which GNPs increased the fracture toughness by 99 percent. At the same time, other similar studies of ceramics toughened by GNPs $[5,16-$ $19,21,23,27,29]$ have not demonstrated such strong toughening.

Structural studies have shown that an increase in the fracture toughness of such nanocomposites is associated primarily with crack bridging by graphene inclusions, the pull-out of graphene inclusions from the matrix, as well as with crack deflec-

Corresponding author: S.V. Bobylev, e-mail: bobylev.s@gmail.com

(C) 2018 Advanced Study Center Co. Ltd. 


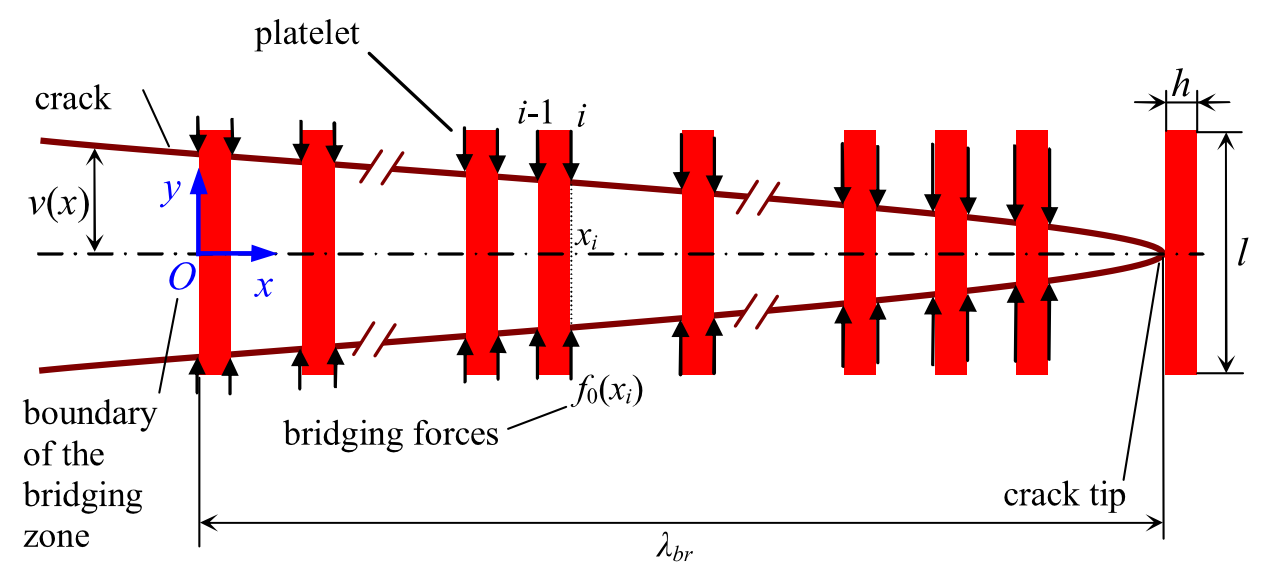

Fig. 1. Crack in a ceramic/graphene composite with aligned graphene platelets. The traction in the crackbridging zone are discretized into a series of concentrated forces $f_{0}\left(x_{i}\right)$.

tion and branching [15-19,22,23], although the presence of graphene wrinkles and out-of-plane compression of graphene platelets can also contribute to the toughening of ceramic/graphene composites [10]. At the same time, the experimental data on fracture toughness of ceramic/graphene composites [3-5,10-36] do not allow one to reveal the effects of the geometry of graphene nanoplatelets and structure of ceramics on the fracture toughness of ceramic/graphene composites.

Several authors [37-39] previously theoretically examined the toughening effects of graphene platelets in ceramic- and polymer-matrix composites. In particular, Zhang et al. [37] estimated the contribution of graphene platelet pull-out to the fracture toughness of a polymer reinforced by graphene platelets. To do so, they calculated the work needed to pull graphene platelets out of the matrix near the crack tip. However, their model seems to be simplified because they assumed that each graphene platelet is completely pulled out immediately after the crack front passes by the platelet, which can be the case only for very short platelets. Besides, they neglected the contribution of platelet bridges in the wake of the crack. Ramirez and Osendi [38] applied the solution [40] describing the toughening of fiber-reinforced ceramics for ceramics reinforced by graphene platelets, which, however, did not account for the real planar shape of platelets. Both studies $[37,38]$ obtained the linear dependence between the critical energy release rate and the volume fraction of graphene.

Chou and Green [41] and Ovid'ko and Sheinerman [39] calculated the effect of crack deflection in ceramics reinforced by $\mathrm{SiC}$ and graphene platelets, respectively. They demonstrated that even at a small volume fraction of platelets (up to $2-4 \%$ ), crack deflection can increase fracture toughness by several tens percent. At the same time, electron microscopy observations (see, e.g., reviews $[4,5,10,11])$ of cracks and fracture surfaces of ceramic/graphene composites highlight a very important role of crack bridging combined with platelet pull-out in the toughening of ceramic/graphene composites. The important role of crack bridging is further supported by the character of crack resistance curves [5] (which demonstrate a significant increase of the fracture toughness with the crack length), the observations of GNP wrinkling $[4,5,10,11]$ and wrapping around grains $[15,42]$, which can strongly increase the friction force between the GNPs and the ceramic matrix in the course of GNP pull-out. Therefore, in the following, we will employ the method developed by Shao et al. [43] for the description of the bridging-induced toughening of nacre to calculate the effect of crack bridging in ceramic/graphene composites on their fracture toughness, depending on the volume fraction and geometric parameters of graphene platelets.

\section{CRACK BRIDGING IN CERAMIC/ GRAPHENE COMPOSITES. MODEL}

Let us consider crack propagation in a deformed composite specimen containing graphene platelets. To do so, consider a model straight semi-infinite mode I crack intersecting a system of identical platelets (with the equal length and width $l$ and thickness $h$ ) perpendicular to the crack plane (Fig. 1). In the region behind the crack tip where the distance between the crack surfaces is smaller than the graphene platelet length $l$, referred to as the crackbridging zone, platelets form bridges between the crack surfaces. The friction between the platelets 

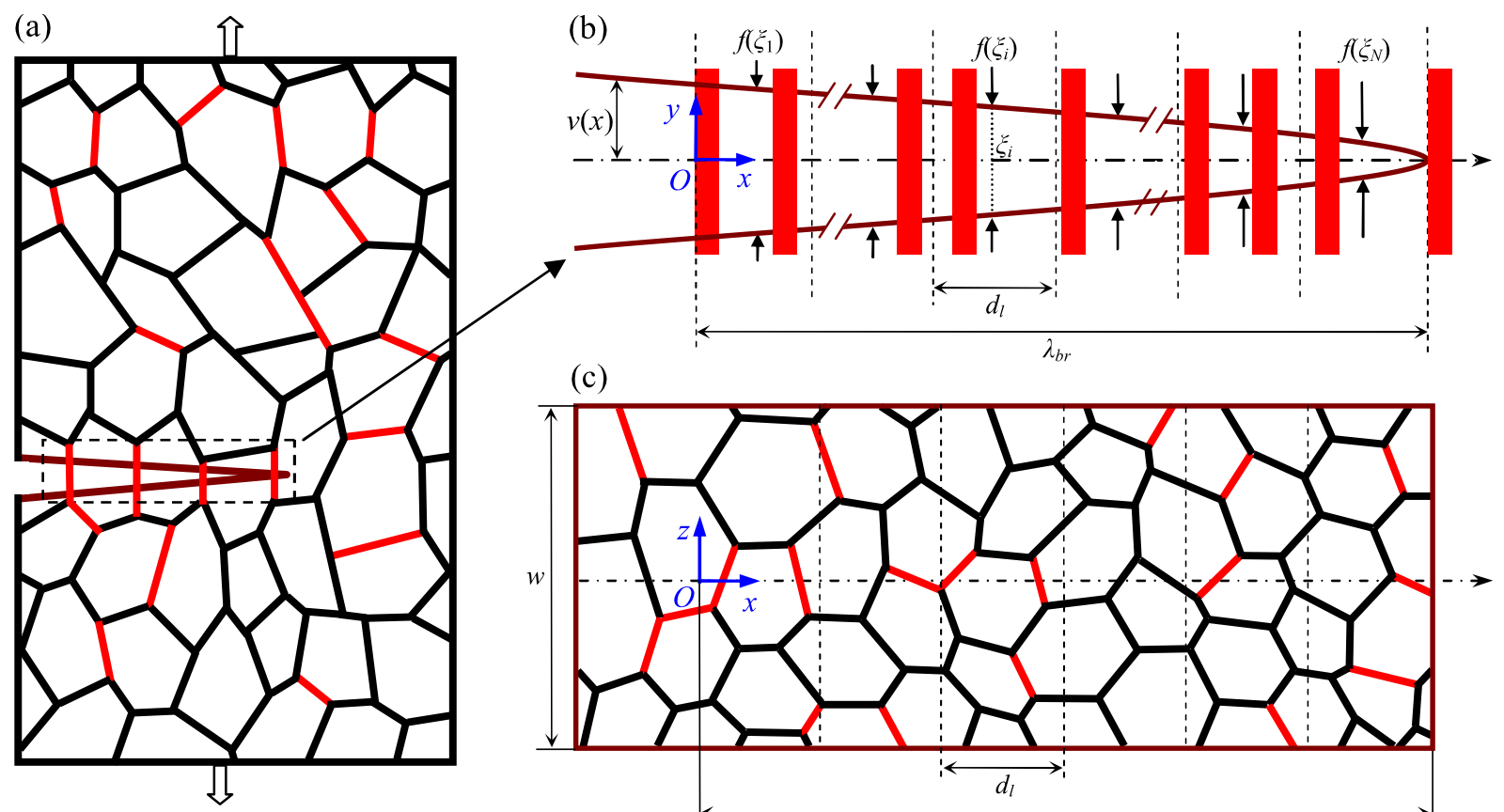

(c)

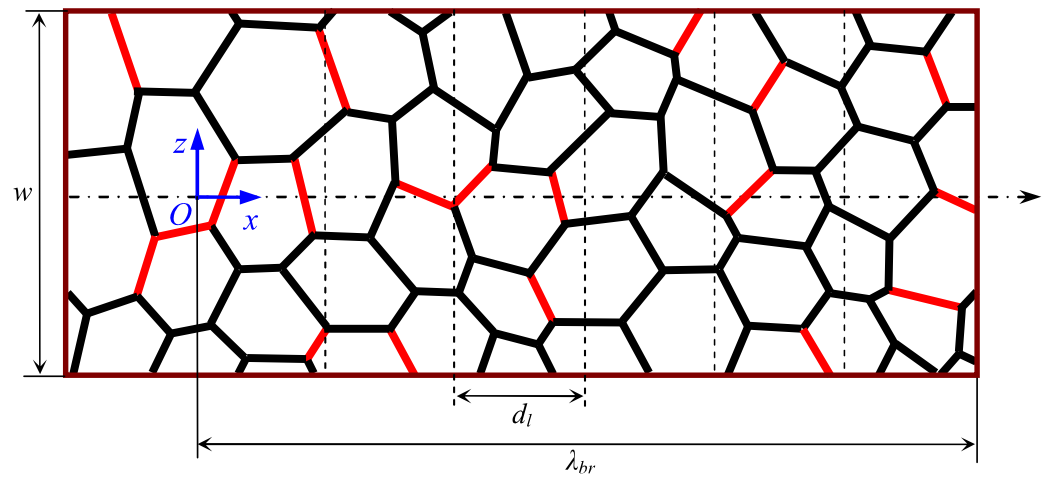

Fig. 2. Geometry of crack bridging in a ceramic/graphene composite. (a) Schematic view of a composite sample with a propagating crack. The red color is used for the grain boundaries occupied by graphene platelets. (b) Enlarged view of the crack bridging zone. (c) Top-down view on the crack plane showing grain boundary structure with a fraction of grain boundaries containing graphene platelets (shown in red).

and the ceramic matrix produces the bridging forces, acting at each matrix/platelet interface (Fig. 1). These forces create a resistance to the crack opening, thereby increasing the fracture toughness of the ceramic/graphene composite. In the Cartesian coordinate system $(x, y)$ with the origin at the boundary of the crack-bridging zone (see Fig. 1) these forces (per unit length in the direction normal to the plane of Fig. 1) are written [43] as:

$$
f_{0}\left(x_{i}\right)=\tau\left(x_{i}\right)\left[\frac{l}{2}-v\left(x_{i}\right)\right] .
$$

Here $x_{i}$ are the coordinates of the matrix/platelet interfaces where the forces act ( $i$ takes the integers from 1 to $N$, where $N$ is the total number of bridging forces), $v\left(x_{j}\right)$ is the crack face opening displacement at the position $x=x_{i}$ (which is equal to the pull-out length of the platelet at the same location), $\tau\left(x_{i}\right)$ is the average shear stress at the interface between the bridging platelet and the matrix. In the following, for the examined case of ceramic/graphene composites, we assume that the stress $\tau\left(x_{i}\right)$ does not depend on the crack opening displacement at the point $x=x_{i}$ and put $\tau\left(x_{i}\right)=\tau_{0}$, where $\tau_{0}$ is a material constant.

One should note that in reality, graphene platelets, which commonly occupy ceramic grain boundaries, do not lie in parallel planes (see Fig. 2a). At the same time, in graphene/ceramic composites produced using spark plasma sintering with uniaxial pressure, graphene platelets tend to predominantly lie in the planes normal or nearly normal to the direction of the pressure applied during sintering (see, e.g., reviews $[4,5,10,11]$ ). (For example, in $\mathrm{Si}_{3} \mathrm{~N}_{4} /$ graphene composites produced in Ref. [44] by spark plasma sintering, more than $80 \%$ of the graphene platelets were oriented within $\pm 15^{\circ}$ of the dominant orientation direction.) Therefore, we focus on the case of ceramic/graphene composites with aligned graphene platelets, subjected to a uniaxial tensile load applied along such platelets, and consider the platelets as lying in the planes normal to the crack plane. The deviations of their orientations from these planes will be incorporated into the parameter $\tau_{0}$. Within our model, this parameter also incorporates the wrinkling and bending of graphene platelets, which increase the friction force between the platelets and the matrix in the course of platelet pull-out, as well as the relaxation of the thermal mismatch stresses during this process. We also assume that in addition to the case of aligned platelets, the above model can be used as a first approximation for the situation of random platelet orientations. In the latter situation, the parameter $\tau_{0}$ should also incorporate the deviations of the plate- 
let orientations from the planes normal to the crack plane.

We further assume that graphene fully covers a fraction $f_{g r}$ of grain boundaries (these boundaries are shown in red in Fig. 2a). To account for the spatial distribution of platelets in the framework of the model approach developed by Shao et al. [43], we use the following procedure. We divide the crack-bridging zone in the crack plane into a system of $N$ identical layers whose width $d_{l}$ in the crack growth direction is small compared to the length $\lambda_{b r}$ of the crack bridging zone (see Fig. $2 \mathrm{~b}$ and the top-down view on the crack plane in Fig. 2c). In this case, the crack face opening displacements $v$ can be considered as constant within each layer. We approximate an ensemble of the concentrated forces acting within each layer at the matrix/platelet interfaces by a single concentrated force $F\left(\xi_{i}\right)$ defined as the sum of the forces created by all the graphene platelets intersecting this layer. We also assume that the forces $F\left(\xi_{j}\right)$ act in the middle section of each layer at the positions given by the coordinates (in the coordinate system shown in Figs. $2 \mathrm{~b}$ and $2 \mathrm{c}$ )

$\xi_{i}=(i-1 / 2) d_{l}, \quad i=1,2, \ldots, N$.

The forces $F\left(\xi_{j}\right)$ can be expressed as

$$
F\left(\xi_{i}\right)=2 \tau_{0}\left[l / 2-v\left(\xi_{i}\right)\right] L_{g b} f_{g r} .
$$

Here the factor $\tau_{0}\left[l / 2-v\left(\xi_{j}\right)\right]$ represents the force (per unit length) given by formula (1), and it is doubled to account for the friction on both lateral surfaces of a platelet, $L_{g b}$ is the total length (in the crack plane) of all the grain boundaries intersecting the crack plane within the ith layer, and the product $L_{g b} f_{g r}$ represents the total length of grain boundaries covered with graphene (i.e. the total length of graphene platelets in the crack plane within the ith layer).

The total length of grain boundary lines within each layer, which is basically the total perimeter of the planar grain boundary structure, can be written as

$$
L_{g b}=\alpha_{1} N_{\text {grains }} d,
$$

where $N_{\text {grains }}$ is the number of grains within the layer, $d$ is the grain size and $\alpha_{1}$ is the constant of the order of unity. Formula (4) reflects the simple geometrical fact that the perimeter of a cross section of a grain by the crack plane is proportional to the grain size. The total number of grains $N_{\text {grains }}$ within each layer can be estimated as the ratio of the layer area to the grain area:

$$
N_{\text {grains }}=\frac{d_{l} w}{\alpha_{2} d^{2}},
$$

where $w$ is the layer length in the direction of the $z$ axis (crack width; see Fig. 2c) and $\alpha_{2}$ is the constant of the order of unity. Then from (4) and (5) we have

$L_{g b}=\frac{\alpha_{1} w d_{l}}{\alpha_{2} d}=\frac{\alpha w d_{l}}{d}$,

where $\alpha=\alpha_{1} / \alpha_{2}$. The parameter $\alpha$ can be easily calculated in the case of regular grain shapes. For example, if we take the grain boundary length as the grain size in the case of model columnar grains with a square base, we get $\alpha=4$, and for columnar grains with the base in the form of regular hexagons, we have $\alpha=4 / \sqrt{3} \approx 2.3$.

The parameter $f_{g r}$ appearing in formula (3) is related to the volume fraction $c$ of graphene, grain size $d$ and platelet thickness $h$ via the following expression:

$$
c \approx \frac{3 h}{d} f_{g r} .
$$

Then, using formulas (3)-(7), we can present the forces $F\left(\xi_{i}\right)$ in the following form:

$$
F\left(\xi_{i}\right)=\frac{2 \alpha c d_{l} w}{3 h} \tau_{0}\left[l / 2-v\left(\xi_{i}\right)\right] .
$$

As a result, the forces per unit length of the layers in the direction of the $z$-axis (see Fig. 2c) are given as

$$
f\left(\xi_{i}\right)=F\left(\xi_{i}\right) / w=\frac{2 \alpha c d_{l}}{3 h} \tau_{0}\left[l / 2-v\left(\xi_{i}\right)\right] .
$$

Notice that the stresses $f\left(\xi_{i}\right)$ do not depend on the grain size.

The fracture toughness $K_{I C}$ of the ceramic/ graphene composite can be written [43] as

$K_{1 C}=K_{1}^{0}-K_{1}^{b r}$,

where $K_{1}^{0}$ is the fracture toughness without the toughening effect of crack-bridging platelets and $K_{1}^{b r}$ is the total stress intensity factor created by the bridging forces, which is negative. The stress intensity factor $K_{l}^{b r}$ is expressed $[43,45]$ as

$$
K_{l}^{b r}=-\sqrt{\frac{2}{\pi}} \frac{\alpha c d_{l} \tau_{0}}{3 h} \sum_{i=1}^{N} \frac{l-2 v\left(\xi_{i}\right)}{\sqrt{\lambda_{b r}-\xi_{i}}} .
$$


The absolute value of $K_{l}^{b r}$ increases with increasing $\lambda_{b r}$, eventually reaching saturation when the crack enters the steady-state propagation mode [43], in which the crack propagates but the crack-bridging zone is kept at a constant length. In the steadystate mode, a new platelet bridge formed at the right end of the crack-bridging zone is always accompanied by a complete pull-out of a platelet at the left end.

In order to use formula (11), one should calculate the crack opening displacements $v\left(\xi_{j}\right)$. This is achieved by solving the following system on $\mathrm{N}$ linear equations [43]:

$$
\begin{aligned}
& v\left(\xi_{i}\right)=\frac{4 K_{i}^{0} \sqrt{\lambda_{b r}-\xi_{i}}}{\sqrt{2 \pi} E}+ \\
& \frac{4 \alpha c d_{l} \tau_{0} \sqrt{\lambda_{b r}-\xi_{i}}}{3 \pi E h} \sum_{n=1}^{N} \frac{l-2 v\left(\xi_{n}\right)}{\sqrt{\lambda_{b r}-\xi_{n}}}- \\
& \frac{2 \alpha c d_{l} \tau_{0}}{3 \pi E h} p \cdot v \cdot \sum_{n=1}^{N}\left[l-2 v\left(\xi_{n}\right)\right] \times \\
& \ln \frac{\sqrt{\lambda_{b r}-\xi_{i}}+\sqrt{\lambda_{b r}-\xi_{n}}}{\left|\sqrt{\lambda_{b r}-\xi_{i}}-\sqrt{\lambda_{b r}-\xi_{n}}\right|}
\end{aligned}
$$

Here $E$ is the Young modulus and p.v. stands for discrete Cauchy principal value (the definition and the instructions on how to calculate p.v. can be found in the Appendix of Ref. [43]). The length of the bridging zone appearing in formula (12) is defined as $\lambda_{b r}=$ $N d_{l}$. For definiteness, we also initially put $d_{l}=3 l$, in which case the relation $d_{l}<<\lambda_{b r}$ should be satisfied, as a rule. (In the latter case $K_{I C}$ should not depend on $d_{l}$.) To calculate the number $N$ of the layers, we solve the system of linear equations (12) for various $N$ until the relation $v\left(\xi_{j}\right) \approx l / 2$ is satisfied (we find value of $N$ that gives $v\left(\xi_{j}\right)$ as close to $l / 2$ as possible). If for the calculated value of $N$, the relation $d_{l}<<\lambda_{b r}$ is not fulfilled, we decrease the value of $d_{l}$ and repeat the calculations. If the solution of system (12) gives $v\left(\xi_{j}\right)<0$ in a small region very close to the crack tip, we put $v\left(\xi_{j}\right)=0$ in this region. After solving equations (12), for specified values of $K_{1}^{0}$ and $\tau_{0}$, we can calculate the fracture toughness $K_{1 c}$ of the ceramic/graphene composite using formulae (10) and (11).

\section{RESULTS AND DISCUSSION}

In this section we calculate the dependences of the fracture toughness of ceramic/graphene composites on various parameters in the exemplary case of yttria stabilized zirconia (YSZ)/graphene compos- ite using the experimental data from Refs. $[29,46]$. All the calculations are performed for the steadystate crack propagation mode characterized by sufficiently large crack lengths (see the previous section). We calibrated our model using experimental data $[29,46]$ for the case of low graphene contents (when fracture toughness is not expected to decay due to increasing porosity typical of the ceramic/graphene composites with a high graphene concentration). For the YSZ/graphene specimens with characteristic graphene platelet dimensions $l=0.5 \mu \mathrm{m}$ and $h=21.7 \mathrm{~nm}$, experimental results [46] are as follows: $K_{1}^{0}=3.84 \mathrm{MPa} \cdot \mathrm{m}^{1 / 2} ; \eta=K_{I C} / K_{1}^{0}$ $=1.083$ for the $Y S Z+0.2 \mathrm{wt}$ \% graphene composite (which gives $c=0.54$ vol. $\%$ using the density values from [46]) and $\eta=1.143$ for the YSZ $+0.3 \mathrm{wt} . \%$ graphene composite (which corresponds to $c=0.81$ vol.\%).

Using the value of the Young modulus $E=577$ GPa for unreinforced YSZ [47] and the values of other parameters specified above, we obtained good fit to experimental data $[29,46]$ at $\alpha=2.5$ (which is close to the value $\alpha \approx 2.3$ for columnar hexagonal grains) and $\tau_{0}=200 \mathrm{MPa}$. In this case, our calculations give: $\eta \approx 1.1$ and 1.15 for $Y S Z+0.2$ wt. $\%$ graphene and $\mathrm{YSZ}+0.3 \mathrm{wt}$ \% graphene, respectively. At high graphene concentrations, however, the calculated toughening ratio $\eta=K_{I C} / K_{l}^{0}$ considerably exceeds the experimental values, which can be attributed to an increase in porosity or the activation of other mechanisms reducing fracture toughness. For example, for $\mathrm{YSZ}+1 \mathrm{wt} . \%$ graphene $(c=2.7$ vol.\%) our model gives $\eta \approx 1.45$ whereas the measured fracture toughness improvement is $\sim 20 \%$.

In Ref. [29] similar YSZ/graphene specimens were produced but with considerably thinner platelets characterized by the average thickness $h=7$ $\mathrm{nm}$. Their average length is not indicated, but SEM images from Ref. [29] demonstrate that the platelets are several hundreds nanometers in length (most probably, in the range $300-500 \mathrm{~nm}$ ). The measurements [29] provided the following results: $K_{1}^{0}=7.8$ $\mathrm{MPa} \cdot \mathrm{m}^{1 / 2}, \eta \approx 1.038$ and 1.09 for $c=0.25$ and 0.5 vol.\%, respectively. For $h=7 \mathrm{~nm}, l=500 \mathrm{~nm}, \alpha=$ 2.5 , and $\tau_{0}=200 \mathrm{MPa}$, our calculations yield: $\eta \approx$ 1.03 at $c=0.25$ vol. $\%$ and $\eta \approx 1.07$ at $c=0.5$ vol. $\%$. In contrast to the results of Ref. [46], for higher graphene concentrations, the experimental data from Ref. [29] shows rapid growth of fracture toughness. For example, at $c=2$ vol.\%, our model gives $\eta \approx$ 1.27 whereas the measured increase in toughness is $\sim 64 \%$.

From the above analysis it follows that the values $\alpha=2.5$ and $\tau_{0}=200 \mathrm{MPa}$ provide fairly good fit 

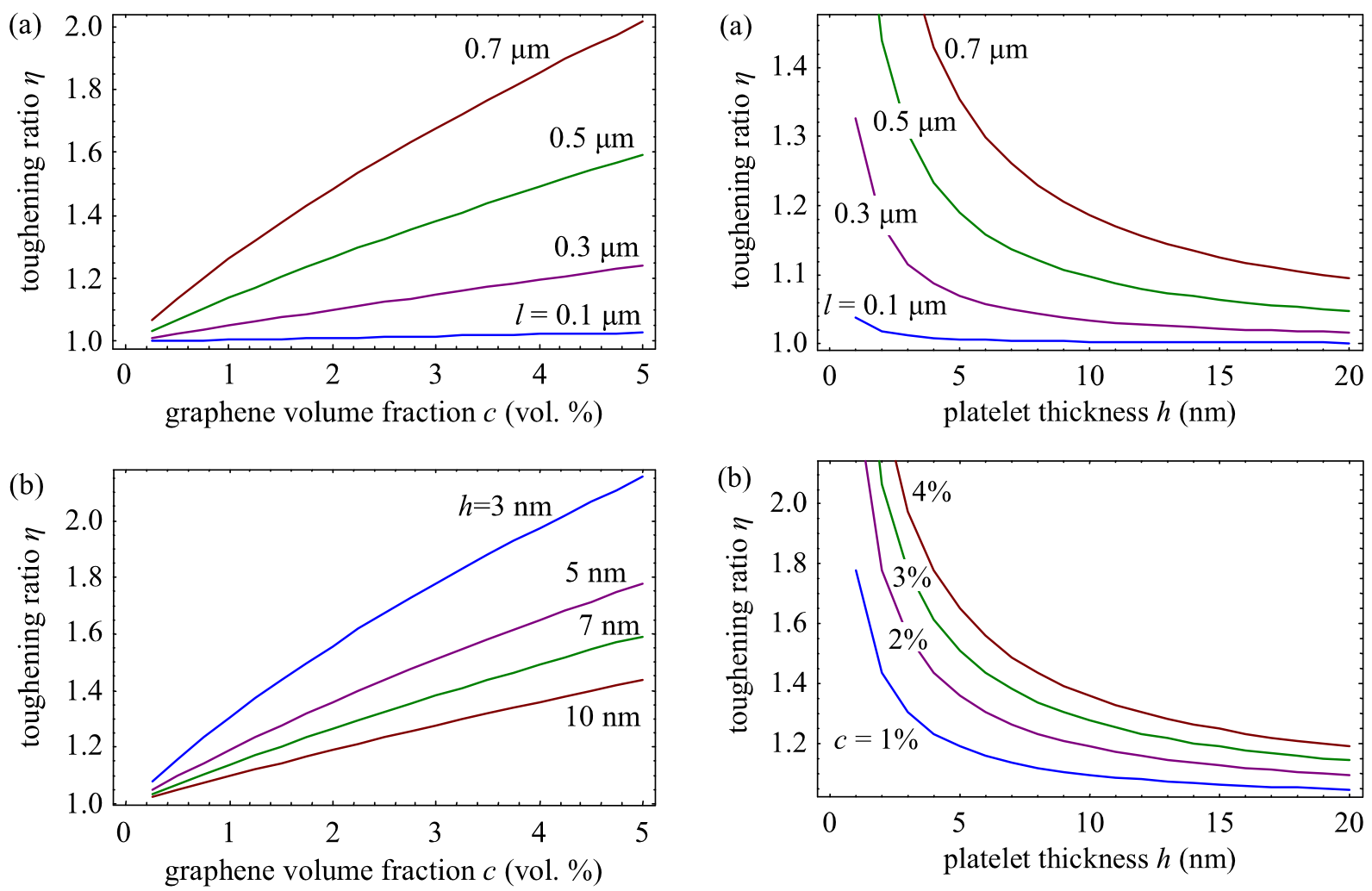

Fig. 3. Dependences of the toughening ratio $\eta$ on the graphene volume fraction $c$ for YSZ/graphene composites with (a) the graphene platelet thickness $h=7 \mathrm{~nm}$ and various values of the platelet length $l$; (b) the graphene platelet length $l=0.5 \mu \mathrm{m}$ and various values of the platelet thickness $h$.

of the model results to the experimental data for the YSZ/graphene system at a low graphene concentration. To further confirm that this value is within the range of realistic values, we have compared it with the interfacial shear strength of $\mathrm{Al} / \mathrm{graphene}$ and polyethylene/graphene obtained from the atomistic simulations (we have not found the data on the interfacial shear strength of YSZ/graphene). The value $\tau_{0}=200 \mathrm{MPa}$ proves to be close enough to the simulated interfacial shear strength of $\mathrm{Al} /$ graphene (up to $183 \mathrm{MPa}$ ) and polyethylene/graphene (up to 232 $\mathrm{MPa}$ ) obtained in Ref. [48]. It also lies in the range of the interfacial shear strength values (from 140 to $760 \mathrm{MPa}$ ) obtained in Ref. [49] for polyethylene/ graphene. This implies that our estimate of $\tau_{0}$ is close enough to the characteristic values of the interfacial shear strength of other composites containing graphene platelets.

Now we can plot the dependences of the toughening ratio $\eta=K_{l} d K^{0}$ on various parameters $(c, l$, $h)$ to obtain theoretical estimates for the crack-bridging induced toughening. Below we put $\alpha=2.5, \tau_{0}=$

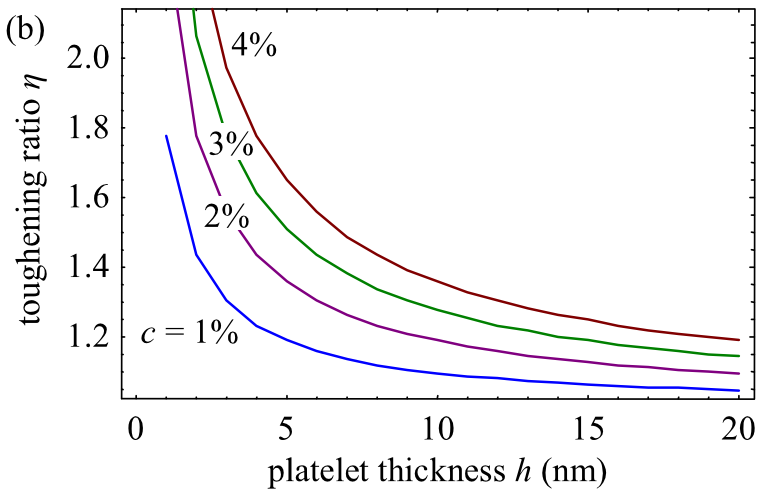

Fig. 4. Dependences of the toughening ratio $\eta$ on the graphene platelet thickness $h$ for YSZ/graphene composites with (a) the graphene volume fraction $c=1 \%$ and various values of the platelet length $l$; (b) the graphene platelet length $l=0.5 \mu \mathrm{m}$ and various values of the graphene volume fraction.

$200 \mathrm{MPa}, E=577 \mathrm{GPa}$ [47], and $K_{1}^{0}=7.8 \mathrm{MPa}$. $\mathrm{m}^{1 / 2}$ [29]. Fig. 3 shows the dependences of the toughening ratio $\eta$ on the graphene volume fraction $c$ for various values of the platelet length $l$ (Fig. 3a) and platelet thickness $h$ (Fig. 3b). The curves in Fig. 3a and $3 \mathrm{~b}$ are calculated for $h=7 \mathrm{~nm}$ and $l=0.5 \mu \mathrm{m}$, respectively. Fig. 3 demonstrates that the toughening ratio $\eta$ increases with increasing the graphene content $c$ in the composite, and the normalized increase in fracture toughness due to graphene $\left(K_{1 C^{-}}\right.$ $\left.K_{1}^{0}\right) / K_{1}^{0}=\eta-1$ scales with the graphene volume fraction $c$ approximately as $\eta-1 \sim c^{0.8}$. This means that our model predicts faster growth of the fracture toughness with the graphene volume fraction than previous models [37,38], which predicted $K_{1 C}-K_{1}^{0} \sim$ $c^{1 / 2}$.

It should be noted that Fig. 3 describes the case of small enough graphene concentration. The experiments on ceramic/graphene composites demonstrate that at sufficiently high graphene concentrations, further increase in cleads to a decrease in toughness attributed to graphene clustering and 

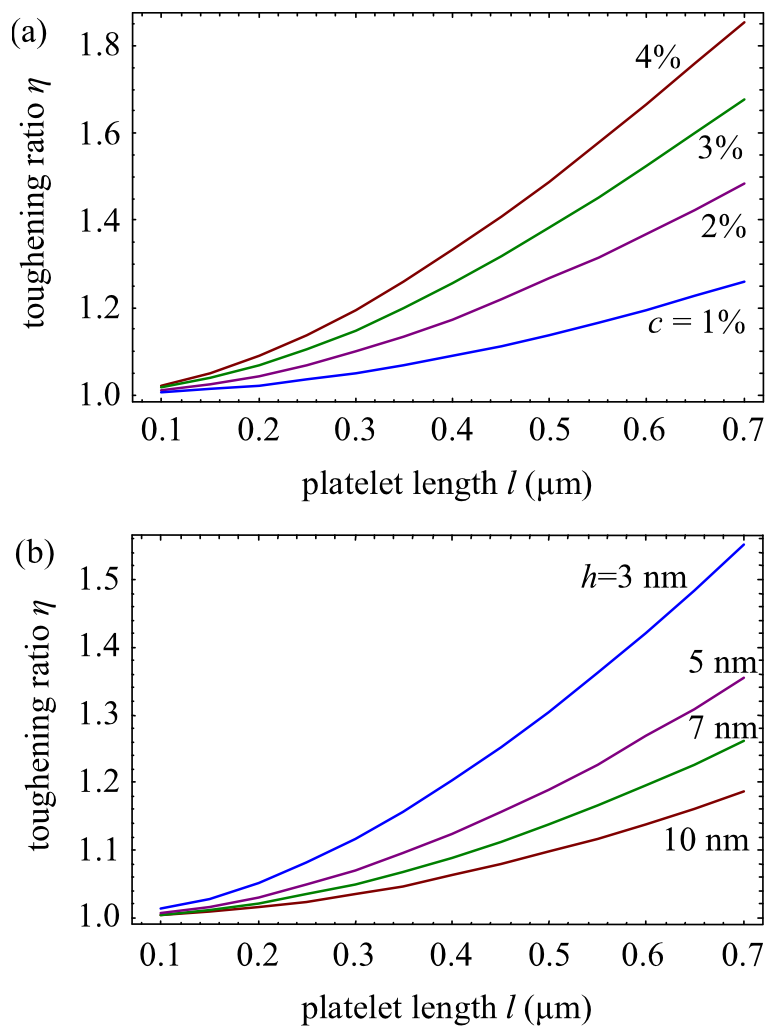

Fig. 5. Dependences of the toughening ratio $\eta$ on the graphene platelet length $l$ for YSZ/graphene composites with (a) the platelet thickness $h=7 \mathrm{~nm}$ and various values of the graphene volume fraction $c$; (b) the graphene volume fraction $c=1 \%$ and various values of the platelet thickness $h$.

porosity increase (e.g., $[4,5,10,11])$. In this case, the dependences shown in Fig. 3 are no more valid.

Fig. 4 shows the dependences of the toughening ratio $\eta$ on the platelet thickness $h$ for various values of the platelet length $l$ (Fig. 4a) and graphene volume fraction $c$ (Fig. 4b). The curves in Figs. $4 \mathrm{a}$ and $4 \mathrm{~b}$ are calculated for $c=1$ vol.\% and $l=0.5$ $\mu \mathrm{m}$, respectively. Fig. 4 shows that the toughening ratio $\eta$ decreases with increasing platelet thickness $h$. The reason is that the higher the thickness of the platelets, the fewer platelets are found in the composite (for a fixed graphene volume fraction), meaning fewer crack bridges. This means that for a specified graphene volume fraction $c$, thinner platelets produce stronger toughening. However, a simple variation of platelet thickness without a change of their number will not affect crack-bridging induced toughening.

Fig. 5 shows the dependences of the toughening ratio $\eta$ on the platelet length $l$ for various values of the graphene volume fraction $c$ (Fig. 5a) and the platelet thickness $h$ (Fig. 5b). The curves in Fig. 4a are calculated for $h=7 \mathrm{~nm}$ and $c=1$ vol. \%. Fig. 5 demonstrates that the toughening ratio $\eta$ increases with increasing platelet length. Although for a fixed graphene volume fraction $c$, a higher platelet length means fewer platelets in the composite, the fact that a longer platelet is much harder to pull out from the matrix due to a larger area of the interface between the platelet and the matrix easily beats the former factor. The dependences in Fig. 5 are valid for platelet lengths below the values at which graphene platelets can rupture. The critical length $l_{c}$ for the destruction of graphene platelets can be estimated from the equation $\tau_{0} l_{c}=\sigma_{m} h$, where $\sigma_{m}$ is the fracture strength of graphene platelets. The latter relation yields: $l_{c} / h=\sigma_{m} / \tau_{0}$. If we estimate the fracture strength of graphene platelets as that of monolayer graphene (from $35 \mathrm{GPa}$ for some specimens of polycrystalline graphene to $130 \mathrm{GPa}$ for pristine graphene [50-52]), we obtain: $l_{c} / h=175-650$. This means that apart from the upper part of the upper curve in Fig. 5b, the curves in Fig. 5 lie in the range where graphene platelets surely do not rupture.

Figs. $3-5$ demonstrate that if crack bridging is the dominant mechanism controlling crack propagation in YSZ/graphene composites, it can increase fracture toughness by up to $100 \%$, depending on the amount of graphene in the composite and platelet dimensions. This toughness improvement is comparable with the typical experimentally observed toughening values $[27,29,46]$ (usually about 20 $60 \%)$.

\section{CONCLUDING REMARKS}

Thus, we have suggested a model describing the effect of crack bridging on the fracture toughness of ceramic/graphene composites. Within the model, a mode I crack propagates normally to flat aligned graphene platelets, whose pullout from the ceramic matrix in the wake of the crack toughens the composite. For the exemplary case of YSZ/graphene composites, we have demonstrated that the crackbridging-induced fracture toughness enhancement $K_{1 C}-K_{1}^{0}$ scales with the graphene volume fraction $c$ approximately as $K_{1 C}-K_{1}^{0} \sim c^{0.8}$. The calculations also revealed that, for a specified graphene volume fraction, longer platelets produce better crack-bridging-related toughening than smaller ones.

At the same time, for a specified volume fraction of graphene platelets, large platelets are characterized by higher average spacing, which can result in a less uniform platelet distribution and thereby reduce the fracture toughness of the composite. In 
addition, if the platelets do not adhere very well to the matrix, long platelets produce weak interfaces that can themselves initiate fracture or promote the propagation of existing cracks. For example, in alumina/graphene composites, an increase in the length of graphene platelets can activate sliding over the alumina/graphene interfaces near crack tips, which was shown to reduce fracture toughness [53]. Thus, the structural design of tough ceramic/ graphene composites requires a simultaneous account for multiple crack propagation mechanisms. This will be the subject of the further investigations of the authors.

\section{ACKNOWLEDGEMENT}

This work was supported by the Russian Science Foundation (grant 18-19-00255).

\section{REFERENCES}

[1] T. Kuilla, S. Bhadha, D. Yao, N.H. Kim, S. Bose and J.H. Lee // Prog. Polymer. Sci. 35 (2010) 1350.

[2] H. Kim, A.A. Abdala and C.W. Macosko // Macromolecules 43 (2010) 6515.

[3] I.A. Ovid'ko // Rev. Adv. Mater. Sci. 34 (2013) 19.

[4] H. Porwal, S. Gresso and M.J. Reece // Adv. Appl. Ceram. 112 (2013) 443.

[5] A. Centeno, V.G. Rocha, B. Alonso, A. Fernandez, C.F. Gutierres-Gonzalez, R. Torrecillas and A. Zurutuza // J. Eur. Ceram. Soc. 33 (2013) 3201.

[6] I.A. Ovid'ko // Rev. Adv. Mater. Sci. 38 (2014) 190.

[7] H.G.P. Kumar and M.A. Xavior // Procedia Engineering 97 (2014) 1033.

[8] Z. Hu, G. Tong, D. Lin, C. Chen, H. Guo, J. Xu and L. Zhou // Mater. Sci. Technol. 32 (2016) 930.

[9] J. Phiri, P. Gane and T.C. Maloney // Mater. Sci. Eng. B 215 (2017) 9.

[10] A. Nieto, A. Bisht, D. Lahiri, C. Zhang and A. Agarwal // Int. Mater. Rev. 62 (2017) 241.

[11] P. Miranzo, M. Belmonte and M.I. Osendi // J. Eur. Ceram. Soc. 37 (2017) 3649.

[12] I.A. Ovid'ko // Philos. Trans. Roy. Soc. A 373 (2015) 20140129.

[13] T. He, J. Li, L. Wang, J. Zhu and W. Jiang // Mater. Trans. 50 (2009) 749.

[14] Y. Fan, L. Wang, J. Li, S. Sun, F. Chen, L. Chen and W. Jiang // Carbon 48 (2010) 1743.
[15] L.S. Walker, V.R. Marroto, M.A. Rafiee, N. Koratkar and E.L. Corral // ACS Nano 5 (2011) 3182.

[16] O. Tapaszto, L. Tapaszto, M. Marko, F. Kern, R. Gadow and C. Balazsi // Chem. Phys. Lett. 511 (2011) 340.

[17] K. Wang, Y. Wang, Z. Fan, J. Yan and T. Wie // Mater. Res. Bull. 46 (2011) 315.

[18] L. Kvetkova, A. Duszova, P. Hvizdos, J. Dusza, P. Kun and C. Balazsi // Scr. Mater. 66 (2012) 793.

[19] J. Lui, H. Yan, M.J. Reece anf K. Jiang // J. Eur. Ceram. Soc. 32 (2012) 4185.

[20] R.J. Young, I.A. Kinloch, L. Gong and K.S. Novoselov // Compos. Sci. Technol. 72 (2012) 1459.

[21] J. Lui, H. Yan and K. Jiang // Ceram. Int. 39 (2013) 6215.

[22] A. Nieto, D. Lahiri and A. Agarwal // Mater. Sci. Eng. A 582 (2013) 338.

[23] H. Porwal, P. Tatarko, S. Grasso, J. Khaliq, I. Dlouhy and M.J. Reece // Carbon 64 (2013) 359.

[24] Y. Fan, M. Estili, G. Igarashi, W. Jiang and A. Kawasaki // J. Eur. Ceram. Soc. 34 (2014) 443.

[25] B. Lee, M.Y. Koo, S.H. Jin, K.T. Kim and S.H. Hong // Carbon 78 (2014) 212.

[26] C. Ramirez, P. Miranzo, M. Belmonte, M.I. Osendi, P. Poza, S.M. Vega-Diaz and M. Terronez // J. Eur. Ceram. Soc. 34 (2014) 161.

[27] J.H. Shin and S.H. Hong // J. Eur. Ceram. Soc. 34 (2014) 1297.

[28] E. Bódis, O. Tapasztó, Z. Károly, P. Fazekas, S. Klébert, A.M. Keszler, K. Balázsi and J. Szépvölgyi // Open Chem. 13 (2015) 484.

[29] J. Liu, H. Guo, Y. Su, L. Wang, L. Wei, G. Yang, Y. Yang and K. Jiang // Mater. Sci. Eng. A 688 (2017) 70.

[30] B. Mukherjee, O.S. A. Rahman, A. Islam, M. Sribalaji and A.K. Keshri // J. Alloys Compd. 727 (2017) 658.

[31] Y. Huang, D. Jiang, X. Zhang, Z. Liao and Z. Huang // J. Eur. Ceram. Soc. 38 (2018) 4329.

[32] Y. Liu, Y. Ai, W. He, W. Chen and J. Zhang // Ceram. Int. 44 (2018) 16421.

[33] C. López-Pernía, C. Muñoz-Ferreiro, C. González-Orellana, A. Morales-Rodríguez, Á. Gallardo-López, and R. Poyato // J. Alloys Compd. 767 (2018) 994. 
[34] N. Obradović and F. Kern // Ceram. Int. 44 (2018) 16931.

[35] Z. Yin, J. Yuan, W. Xu, M. Chen, S. Yan and Z. Wang // Ceram. Int. 44 (2018) 20299.

[36] H. Zou, Y. Zhang, L. Liu, L. Shi and W. Li // Adv. Appl. Ceram. 117 (2018) 420.

[37] L. Zhang, X.G. Zhang, Y. Chen, J.N. Su, W.W. Liu, T.H. Zhang and Y.T. Wang // Appl. Phys. Lett. 105 (2014) 161908.

[38] C. Ramirez and M.I. Osendi // Ceram. Int. 40 (2014) 11187.

[39] I.A. Ovid'ko and A.G. Sheinerman // Rev. Adv. Mater. Sci. 43 (2015) 52.

[40] G.H. Campbell, M. Rühle, B.J. Dalgleish and A.G. Evans // J. Am. Ceram. Soc. 73 (1990) 521.

[41] Y.S. Chou and D.J. Green // J. Am. Ceram. Soc. 76 (1993) 1985.

[42] I. Ahmad, M. Islam, H.S. Abdo, T. Subhani, K.A. Khalii, A.A. Almajid, B. Yazdani and Y. Zhu // Mater. Des. 88 (2015) 1234.

[43] Y. Shao, H.-P. Zhao, X.-Q. Feng and H.Gao // J. Mech. Phys. Solids 60 (2012) 1400.

[44] O. Tapasztó, L. Tapasztó, H. Lemmel, V. Puchy, J. Dusza, C. Balázsi and K. Balázsi // Ceram. Int. 42 (1002) 2016.
[45] B. Budiansky and J.C. Amazigo // J. Mech. Phys. Solids 37 (1989), 93.

[46] K. Markandan, M.T.T. Tan, J. Chin and S.S. Lim // Ceram. Int. 41 (2015) 3518.

[47] I.D. Muhammad, M. Awang and O. Mamat // Adv. Mater. Res. 845 (2014) 387.

[48] S.J. Chen, C.Y. Li, Q. Wang and W.H. Duan // Carbon 114 (2017) 557.

[49] M.C. Wang, Z.B. Lai, D. Galpaya, C. Yan, N. Hu and L.M. Zhou // J. Appl. Phys. 115 (2014) 123520.

[50] C. Lee, X. Wei, J.W. Kysar and J. Hone // Science 321 (2008) 385.

[51] P.Y. Huang, C.S. Ruiz-Vargas, A.M. van der Zande, W.S. Whitney, M.P. Levendorf, J.W. Kevek, S. Garg, J.S. Alden, C.J. Hustedt, Y. Zhu, J. Park, P.L. McEuen and D. A. Muller // Nature 469, 389 (2011).

[52] C.S. Ruiz-Vargas, H.L. Zhuang, P.Y. Huang, A.M. van der Zande, S. Garg, P.L. McEuen, D.A. Miller, R.C. Hennig and J. Park // Nano Lett. 11 (2011) 2259.

[53] H. Porwal, R. Saggar, P. Tatarko, S. Grasso, T. Saunders, I. Dlouhý and M.J. Reece // Ceram. Int. 42 (2016) 7533. 